\title{
3D host cell and pathogen-based bioassay development for testing anti- tuberculosis (TB) drug response and modeling immunodeficiency
}

https://doi.org/10.1515/bmc-2021-0013

received May 20, 2021; accepted June 24, 2021.

\begin{abstract}
Tuberculosis (TB) is a global health threat that affects 10 million people worldwide. Human Immunodeficiency Virus (HIV) remains one of the major contributors to the reactivation of asymptomatic latent tuberculosis (LTBI). Over the recent years, there has been a significant focus in developing in-vitro 3D models mimicking early events of Mycobacterium tuberculosis (Mtb) pathogenesis, especially formation of the granuloma. However, these models are low throughput and require extracellular matrix. In this article, we report the generation of a matrix-free $3 \mathrm{D}$ model, using THP-1 human monocyte/macrophage cells and mCherryexpressing Mycobacterium bovis BCG (Bacilli Camille Guérin), henceforth referred as 3D spheroids, to study the host cell-bacterial interactions. Using mCherry-intensitybased tracking, we monitored the kinetics of BCG growth in the 3D spheroids. We also demonstrate the application of the 3D spheroids for testing anti-TB compounds such as isoniazid (INH), rifampicin (RIF), as well as a host-directed drug, everolimus (EVR) as single and combinational treatments. We further established a dual infection 3D spheroid model by coinfecting THP-1 macrophages with BCG mCherry and pseudotype HIV. In this HIV-TB
\end{abstract}

*Corresponding author: Biju Parekkadan, Department of Biomedical Engineering, Rutgers, The State University of New Jersey, NJ 08854; Department of Medicine, Rutgers Biomedical Health Sciences,

Rutgers, The State University of New Jersey, NJ 08854;

E-mail: biju_parekkadan@rutgers.edu

Shilpaa Mukundan, Rachana Bhatt, John Lucas, Matthew Tereyek, Department of Biomedical Engineering, Rutgers, The State University of New Jersey, NJ 08854

Theresa L. Chang, Selvakumar Subbian, Public Health Research Institute, New Jersey Medical School, Rutgers, The State University of New Jersey, NJ 07103

\#Equal contribution co-infection model, we found an increase in BCG mCherry growth within the 3D spheroids infected with HIV pseudotype. The degree of disruption of the granuloma was proportional to the virus titers used for co-infection. In summary, this 3D spheroid assay is an useful tool to screen anti-TB response of potential candidate drugs and can be adopted to model HIV-TB interactions.

Keywords: Bioassay; Tuberculosis; Drug response; Human Immunodeficiency Virus; HIV-TB co-infection; granuloma; anti-tuberculosis drugs.

\section{Introduction}

Tuberculosis (TB) is disseminated through aerosols generated from a person with active disease to a healthy individual. One-fourth of the world's population is thought to be infected with Mycobacterium tuberculosis (Mtb), yet only $5-10 \%$ of the population develops active TB while the rest develops mostly asymptomatic latent infection (LTBI) [1,2]. The host immune system forms the first line of defense by forming granulomatous structures to contain the infection. Mtb can remain latent in these granulomas for decades and reactivate with immunosuppression, for example, during human immunodeficiency virus (HIV) infection. HIV infections contribute to around 770,000 deaths worldwide. About 251,000 deaths in TB patients are linked to TB-HIV co-infections (CDC,2018) [3]. The risk of LTBI reactivation in HIV-positive patients is 20-fold higher when compared to HIV-negative patients [4]. This makes HIV co-infection one of the largest causes of death in TB patients. However, our understanding of what happens to TB granulomas upon HIV co-infection is very limited at present.

Currently, to study the disease progression and identify new therapies, HIV-Mtb co-infection models have been established using small animals and non-human primates 
(NHP). Humanized mouse models demonstrate critical features of TB granulomas such as necrosis [5] and can also be infected with HIV [6]. Similarly, NHP infected with Simian Immunodeficiency Virus (SIV) and Mtb also show close similarity to human pathology [7-9]. However, these in vivo models are expensive, low throughput and take a long time to create. In addition, there is a lack of tools to monitor the interaction of host and pathogen in real-time. Therefore, there is a need to develop simple in vitro tools to study host-pathogen interactions in HIV-Mtb co-infections.

Tremendous progress has been made recently for generating in vitro model systems to study $\mathrm{TB}$ granulomas. In vitro assays to screen anti-TB compounds use macrophage cell lines and freshly isolated peripheral blood mononuclear cells (PBMCs) cultured in 2D or as cellular aggregates (3D) [10]. The 3D aggregate models using PBMCs mimic the TB granuloma structure by incorporating heterogeneous cell types of the human TB pathology [11-16]. Engineering mycobacteria with florescent reporters have also been combined with 3D aggregate assays to monitor bacterial cell survival and killing in real-time [17-20]. These technical advances in modeling TB granulomas in 3D and engineering bacilli have led to significant advances in our understanding of host-pathogen interactions in the granuloma, TB pathology, LTBI and reactivation of disease with immunomodulatory therapeutic compounds [10,21-25]. For example, Kapoor et al demonstrated that treatment of Mtb infected 3D PBMC aggregates with tumor necrosis factor-alpha (TNF- $\alpha$ ) inhibitor increase the metabolic activity of Mtb, when compared to control IgG treated granulomas [26]. Similarly, Mezouar et al. used Sepharose beads coated with mycobacterial proteins treated with etanercept, a monoclonal antibody-based inhibitor for TNF- $\alpha$ to demonstrate that granuloma formation was not impacted by TNF- $\alpha$ blockade. Furthermore, etanercept prevented the formation of multi-nucleated giant macrophages and caused an increase in M2 polarization cytokines, such as IL-10 and IL-17 [25]. Similar to the TNF- $\alpha$ blockade, the checkpoint inhibitor for PD-1 (program cell death protein-1) is also known to increase the risk for reactivation of $\mathrm{TB}$ in patients. Tereza et al demonstrated the increase in mycobacterial proliferation within 3D PBMC aggregates, upon treatment with PD-1 inhibitor [27]. These reports demonstrate that LTBI reactivation can be recapitulated using 3D aggregate system. However, none of these models have been adapted to study HIV-Mtb co-infections. Therefore, there is an unmet need to develop an in-vitro granuloma mimetic platform to study HIV-Mtb co-infections and understand host-pathogen interactions within an HIV active granuloma.
Mtb and HIV co-infection constitute a symbiotic environment for both the pathogens [28, 29]. Their co-infection worsens the prospect for the host immune system to fight both these infections. Although, antiretroviral therapy (ART) regimens have greatly reduced HIV-associated morbidity and mortality, concurrent treatment and management of HIV/TB is a huge concern [30-32], particularly in endemic countries. Given the enormous prevalence of LTBI cases and no vaccine available against HIV, the HIV-TB remains a field of significant scientific challenge that needs to be addressed by integrating the advances in disease modeling from the TB field to study HIV-Mtb interactions.

To address this gap in the in vitro model development, we describe a simple bio-engineered 3D THP-1 monocyte/ macrophage-based 3D spheroid system infected with mCherry-expressing Bacillus Camille Guérin (BCG), as a model of Mtb infection. We demonstrate the applicability of the system to study TB pathogenesis and to test anti-TB compounds such as isoniazid (INH), rifampicin (RIF), as well as a host-directed drug, everolimus (EVR). Further, we adapted this model to study HIV/TB by using pseudotypeHIV to infect 3D granulomas. We demonstrate that this bioengineered 3D co-infection model can be tailored to produce TB-HIV granulomas of tunable sizes. Thus, our report addresses some of the limitations of existing in vitro HIV-TB models using bioengineered high throughput spheroid platform technology.

\section{Methods}

Host cell culture. THP-1 human monocyte cells were cultured in RPMI media (Gibco) supplemented with $10 \%$ fetal bovine serum (FBS) and $1 \%$ penicillin and streptomycin (Gibco). The cells were incubated in $5 \% \mathrm{CO}_{2}$ supply at $37^{\circ} \mathrm{C}$.

Bacterial Strains. Cultures of recombinant Bacillus Calmette-Guérin expressing mCherry (BCG-mCherry), were grown in Middlebrooks $7 \mathrm{H} 9$ broth with $0.05 \%$ tween 80 and $10 \%$ oleic acid-albumin-dextrose-catalase enrichment (OADC) at $37^{\circ} \mathrm{C}$ under continuous shaking to $\log$ phase. The cultures were aliquoted and stored frozen at $-80^{\circ} \mathrm{C}$ until use. Bacterial $\mathrm{CFU} / \mathrm{ml}$ was determined by plating serial dilutions of multiple stocks on Middlebrook $7 \mathrm{H} 10$ agar plates and incubating at $37^{\circ} \mathrm{C}$ for $4-6$ weeks. These stocks were later used for infection of spheroids at multiplicities of infection (MOI) ranging from 0-100 using a volume of 0-10 $\mu \mathrm{L}$.

Spheroid assay. The 96 well Ultralow attachment plates (Corning) were surface treated with Anti-adherence 
rinse solution (Stemcell Tech) for $1 \mathrm{~h}$. Following this, the wells were washed with DPBS. THP-1 cells were seeded at a density of 10,000 cells/ well. The cells were centrifuged at $1300 \mathrm{rpm}$ for $5 \mathrm{~min}$, and the bacterial suspension volume was varied from $0-10 \mu \mathrm{L} /$ well (translating to a multiplicity of infection 0-100). For the activation of spheroids, $100 \mathrm{ng} /$ $\mathrm{ml}$ Phorbol 12-myristate 13-acetate (PMA) containing RPMI media was added to the wells and treated for $24 \mathrm{~h}$ and then switched to standard RPMI media without PMA. Media change of $70 \%$ fresh growth media was performed every day.

HIV infection. HIV-TB co-infected spheroids were generated using pseudotyped HIV pre-infected THP-1 cells and $5 \mu \mathrm{L}$ of BCG mCherry in a 96 well assay format as described above. Briefly, pseudotype luciferaseexpressing HIV virus was produced in HEK293T cells by co-transfection of plasmid encoding envelope-deficient HIV NL4-3 virus and luciferase reporter gene (pNL-LucR-E; provided by N. Landau, AIDS Research \& Reference Reagent Program, National Institute of Allergy and Infectious Disease, National Institutes of Health) along with a plasmid encoding HIV-1 JR-FL Env gp160 or VSV-G as described previously [33]. For infection, HIV-pseudotyped virus and THP-1 cells were mixed in $10 \mu \mathrm{g} / \mathrm{ml}$ polybrene containing RPMI media without serum and antibiotics. The cell-viral suspension was centrifuged at $2400 \mathrm{rpm}$ for $2 \mathrm{~h}$ at $37^{\circ} \mathrm{C}$ followed by additional $2 \mathrm{~h}$ incubation under static condition at $37^{\circ} \mathrm{C}$. The pellet was then washed and plated for $72 \mathrm{~h}$ in complete RPMI before using for co-infection experiments. Luciferase activity of infected THP-1 (in relative light units, RLUs) was analyzed using Pierce firefly luciferase glow assay kit and measured by Varioskan lux plate reader (ThermoScientific) to validate virus titers for the infectivity control.

\section{Drug treatment}

Seventy-two hours post-seeding, spheroids were treated with drugs to measure their therapeutic effect. This is accomplished by removing $50 \%$ of existing spheroid media $(50 \mu \mathrm{L})$ and replacing with $50 \mu \mathrm{L}$ fresh media with $2 \mathrm{X}$ desired drug concentration. Anti-TB compounds isoniazid (INH) and rifampicin (RIF), and everolimus (EVR) were purchased from Sigma Aldrich, USA. We also tested dual treatments and triple treatment combinations, making for 7 different drug combinations ( $\mathrm{n}=3$ replicates for each MOI). DMSO-treatment was used as control $(n=3)$. The final concentrations of $t$ drugs used are as follows : INH single treatment $(1.25 \mu \mathrm{M})$, RIF single treatment (0.195 $\mu \mathrm{M})$, EVR single treatment (1 nM), INH+RIF dual treatment (0.625 $\mu \mathrm{M}$ INH and $0.195 \mu \mathrm{M}$ RIF), RIF+EVR dual treatment
(0.195 $\mu \mathrm{M}$ RIF+1 nM EVR), INH+EVR dual treatment ( 0.625 $\mu \mathrm{M}$ INH+1 nM EVR),INH+RIF+EVR triple treatment (0.625 $\mu \mathrm{M}$ INH+0.195 $\mu \mathrm{M}$ RIF $+1 \mathrm{nM}$ EVR) and 0.5\% (v/v) DMSO control. We chose the above drug concentrations based on previously published studies.

The spheroids were treated with the $\operatorname{drug}(\mathrm{s})$ for 48 hours, with images being taken at the 0,24 and 48hour post infection. The mCherry intensity in the spheroids infected with BCG mCherry were measured using Celigo (Nexcelom Biosciences, MA) as described in the following section.

\section{High content fluorescence imaging}

a). mCherry reporter tracking. Intracellular BCG mCherry intensity was tracked by imaging cellular spheroids using the Celigo Image cytometer (Nexcelom Biosciences). Briefly, the spheroids were focused in the bright field channel using Image-Based autofocus. Next, the red channel was used for tracking mCherry intensity. The $z$ position offset was chosen to ensure optimal capture of red fluorescence. The exposure time was optimized and selected based on using MOI 0 group for control (no fluorescence) and BCG infected spheroids (MOI 10-100) for the presence of red fluorescence. Finally, the spheroids were imaged using Celigo software. Automated image analysis was performed to select the outline of spheroids and select mCherry expressing regions within these spheroids. Finally, the quantitative measurements for mean fluorescent intensity were recorded and used for data analysis.

b). Spheroid size tracking. As mentioned in the mCherry reporter tracking section, the same process was carried out for measuring the area of spheroids. In terms of quantitative measurements, the Celigo software (Nexcelom Biosciences) uses the outline of the spheroids for estimating the area. The measurements were recorded and then used for further analysis.

\section{Fluorescence staining and imaging}

a). Live-Dead assay. Viability of THP-1 spheroids was determined using Live-Dead cell viability assay according to the manufacturer's recommended protocol (Invitrogen/ ThermoFisher Scientific). The THP-1 spheroids were rinsed with 1X Dulbecco's Phosphate Buffer Saline (DPBS) and incubated with Calcein AM $(2 \mu \mathrm{M})$ and ethidium homodimer $(4 \mu \mathrm{M})$ solution for 30 minutes. Following this, they were imaged using Celigo (Nexcelom Biosciences). 
b). $\mathbf{p H}$ gradient determination. The intracellular $\mathrm{pH}$ across the spheroids was detected using the pHrodo green stain (Thermofisher) according to the manufacturer's protocol. Briefly, $10 \mu \mathrm{L}$ of the pHrodo dye was added to $100 \mu \mathrm{L}$ of PowerLoad concentrate to form the pre-mix. The pre-mix was then diluted in $10 \mathrm{ml}$ of PBS to form the staining solution. $100 \mu \mathrm{L}$ of the staining solution is added into each well and then incubated for $30 \mathrm{~min}$ at room temperature. Following this, the spheroids were imaged in Celigo (Nexcelom). The GFP intensity at every pixel of each image was recorded using Celigo Image Cytometer (Nexcelom Biosciences,MA) and used for image analysis using the Celigo software.

c). Autophagy. Presence of autophagy in spheroids was assessed using the CYTOID autophagy detection kit according to the manufacturer's protocol (Enzo Life Sciences). Briefly, $2 \mu \mathrm{L}$ of CYTOID green reagent and 1 $\mu \mathrm{L}$ Hoechst were diluted in $1 \mathrm{~mL}$ of $1 \mathrm{x}$ Assay buffer. 100 $\mu \mathrm{L}$ of staining solution was added to each well, and the spheroids were incubated for $30 \mathrm{~min}$ at room temperature. Following this, the spheroids were washed with $1 \mathrm{x}$ Assay buffer thrice and imaged using Axiozoom microscope (Zeiss).

Statistical analysis. GraphPad Prism version 8 was used for data analysis. One-way Anova, Two-way Anova and Tukey post hoc test were used for determining statistical significance. $\mathrm{p}<0.05$ was considered statistically

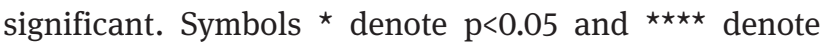
$\mathrm{p}<0.001$.

Ethical approval: The conducted research is not related to either human or animals use.

\section{Results}

\section{Creating a TB spheroid model}

Mtb infection was modeled using a 96 well U-bottom plate as shown in schematic of Fig1a. TB spheroids were generated using THP-1 human monocytes by tuning cell numbers per well ranging from 10,000-50,000 cells/well (Fig 1b). When infecting spheroids with BCG mCherry with an increasing multiplicity of infection (MOI) ranging from 0 (control-no infection) to 100 , we observed an increase in the size of the spheroids (Fig 1c). The spheroid images were then analyzed using Celigo image analysis software (Nexcelom Biosciences, MA) for quantitative size in terms of spheroid area $\left(\mu \mathrm{m}^{2}\right)$ (Fig 1d), mean fluorescent intensity of Calcein AM (host cell viability, Fig 1e) and BCG
mCherry (bacterial viability, Fig 1f). Correlating with the quantitative images in Fig 1d, we observed that there is an increase in spheroid area quantified using the image analysis software, for increasing MOIs. In terms of hostcell viability, we did not observe any change in Calcein AM intensity across different MOIs (Fig 1e). As expected, BCG mCherry intensity increases with the increase in MOI of BCG mCherry (Fig 1f).

\section{Evaluating anti-TB drug response in infected 3D spheroids}

Currently, INH and RIF are the most potent first-line anti-TB drugs, that is administered either individually or in combination for treating LTBI and TB patients, respectively [34]. Over the years, there has been significant research performed using in vitro hollow fiber bioreactor models and in vivo models such as mice to study combinational anti-TB drug response $[35,36]$. Grosset et al observed that combination treatment of INH, RIF and pyrazinamide (PZA) increased bactericidal activity during a 6-month study. However, after an additional 6 months without treatment, they noticed that the relapse rate was higher in the INH-RIF-PZA group when compared to the RIF-PZA combinations confirming antagonism between INH and RIF-PZA chemotherapy [36]. Similarly, Genestet et al demonstrated that INH and RIF combination treatment has a synergistic effect on killing mycobacteria [37]. In a recent study, by Ashley et al, EVR, an mTOR inhibitor, has been shown to demonstrate bactericidal activity in PBMC aggregates infected with Mtb and has synergistic effects when co-treated with INH and PZA [37]. To determine the applicability of this 3D spheroid bioassay for estimation of anti-TB drug response, we treated the spheroids with INH(1.25 $\mu \mathrm{M}), \operatorname{RIF}(0.195 \mu \mathrm{M}), \operatorname{EVR}(1 \mathrm{nM})$ and combination of INH, RIF and EVR (0.625 INH $\mu$ M, $0.195 \mu \mathrm{M}$ RIF, 1nM EVR. We chose the concentration of INH, RIF and EVR based on previously published studies [39-42]. For instance, in a recent study by Ashley et, at a concentration of $1 \mathrm{nM}$ EVR showed a decrease in the number of bacterial colony forming units (CFU) of Mtb Erdman strain [38]. For INH and RIF, the minimum inhibitoty concentration-50 (MIC50) ranges from for 0.1 to $5.8 \mu \mathrm{M}$ for INH [39-41], and $0.05-1 \mu \mathrm{M}$ for RIF, depending on the nature of bacterial strain [41]. Thus, the concentration range chosen for our study is within the limits specified in previously published studies [39-42].

We tracked the response to anti-TB treatment using 3 parameters, namely image- based qualitative profiling 

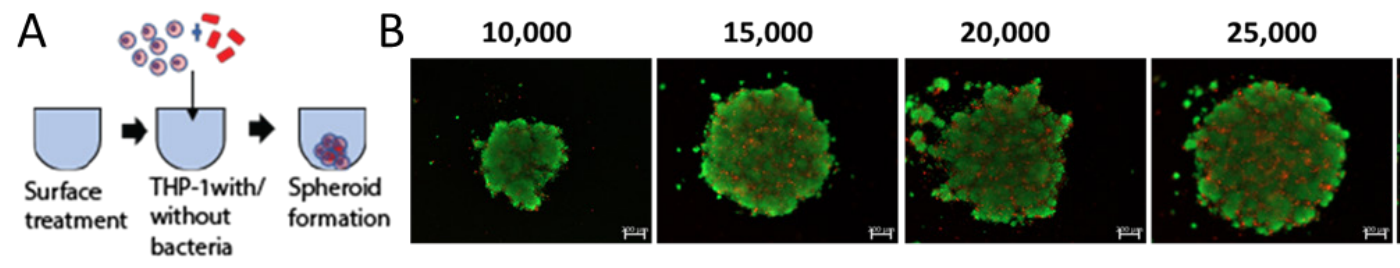

50,000

C

MOl
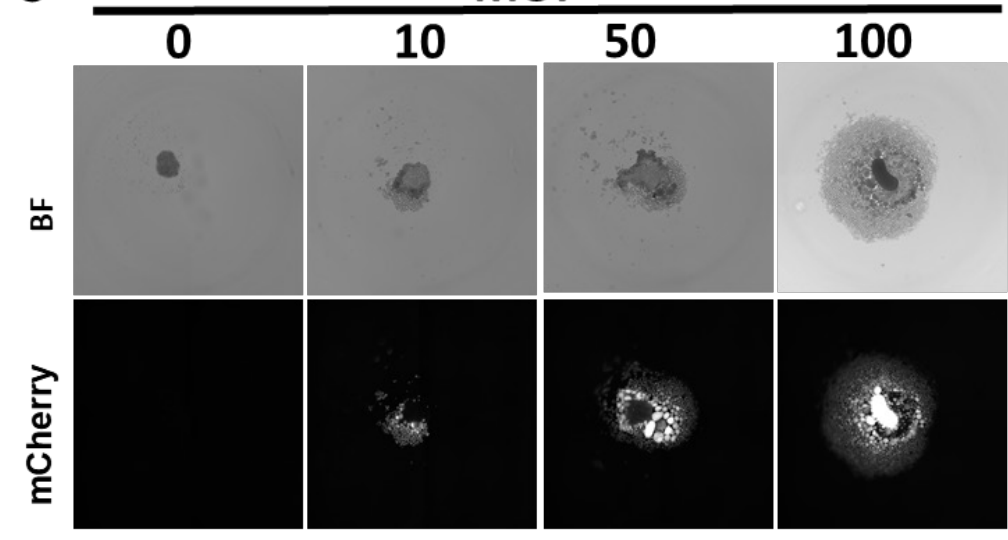

D

$\mathrm{E}$

$\mathrm{F}$
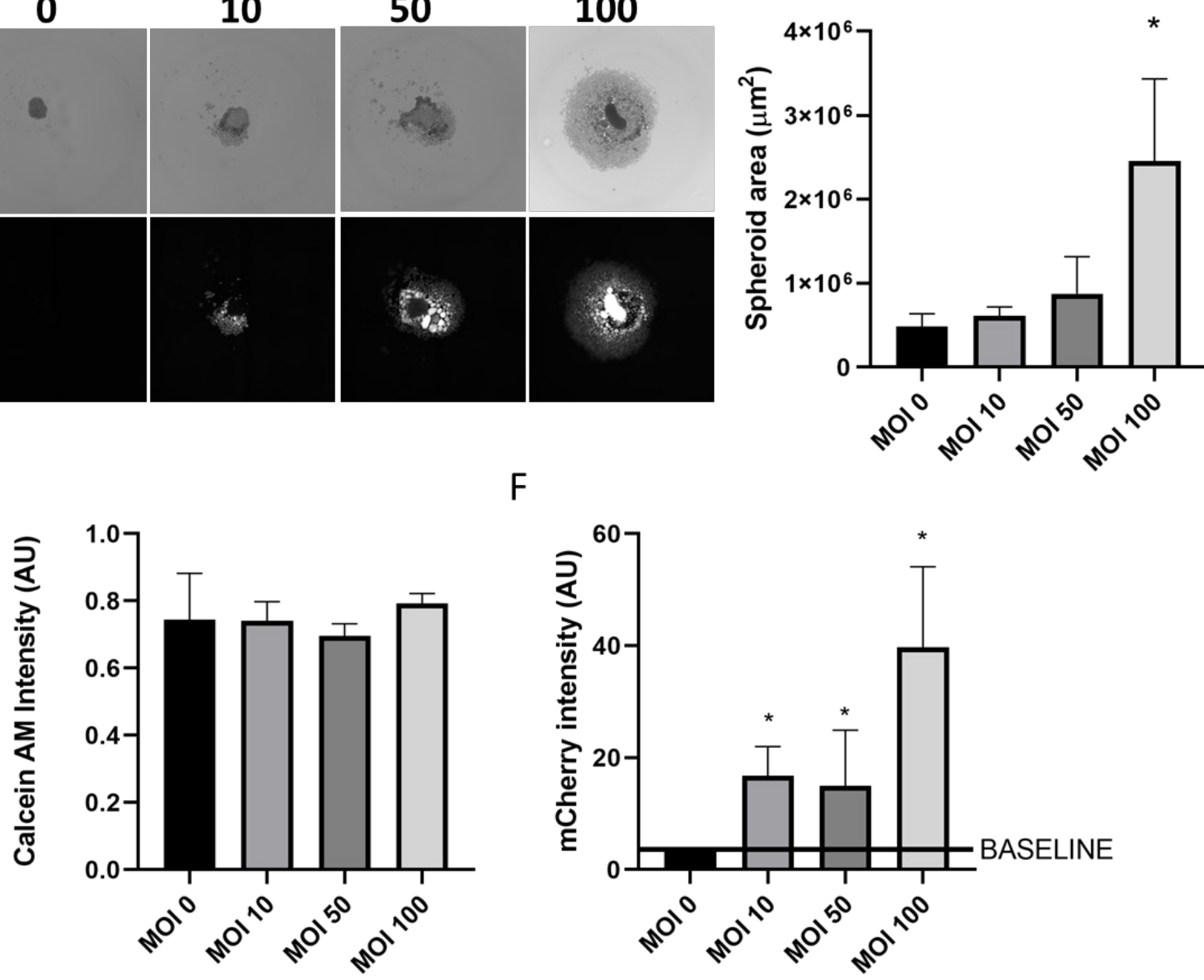

Figure 1: Formation of 3D spheroids and modeling mycobacterial infection. (a) Schematic of spheroid formation in 96 well U-bottom plate (b) Live dead staining of THP-1 cells seeded by varying cell number/well from 10,000-50,000 cells/well (c) Bright field and mCherry channel images of BCG mCherry infected spheroids infected with a varying multiplicity of infection (0-100). Quantification of spheroid area (d), Calcein AM intensity (e) and mCherry intensity (f) of spheroids infected with BCG mCherry at varying MOIs. * denotes statistical significance when compared to $\mathrm{MOI} 0$ group at $\mathrm{p}<0.05$ using one-way Anova and Tukey post hoc test. ( $\mathrm{n}=5$ spheroids/condition)

(Fig 2c), quantitative estimation of BCG mCherry (Fig 2a) and size of TB spheroids (Fig 2b). We observed that combinational drug treatment leads to a decrease in BCG mCherry intensity when compared to single treatment (Fig 2a). Interestingly, we found that with combinational drug treatment with EVR, there is a reduction in size of the spheroids (Fig 2b). We also observed that there is a reduction of spheroid size with EVR treatment (FigS1 Fig 2). Since autophagy is one of the key mechanisms involved in Mtb clearance by host cells [42], we probed the spheroids with autophagy markers. We observed induction of autophagy in BCG mCherry-infected spheroids with drug treatment, especially in EVR treated group (Fig 2c). It has been well reported that the mTOR 
A

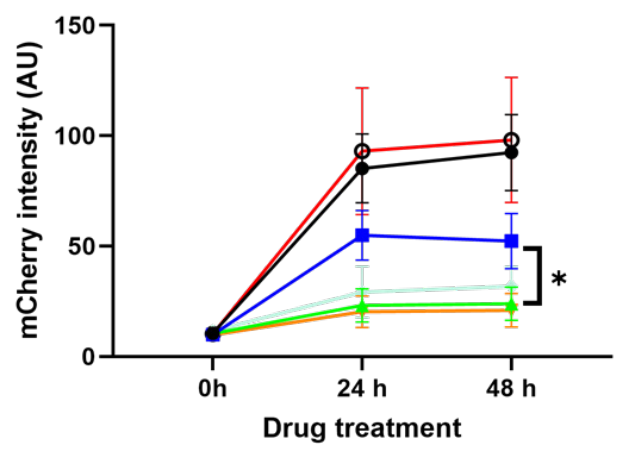

B

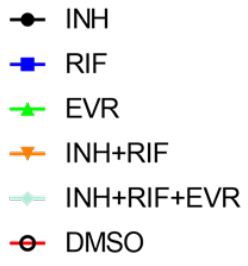

MOI $\square 1$
B

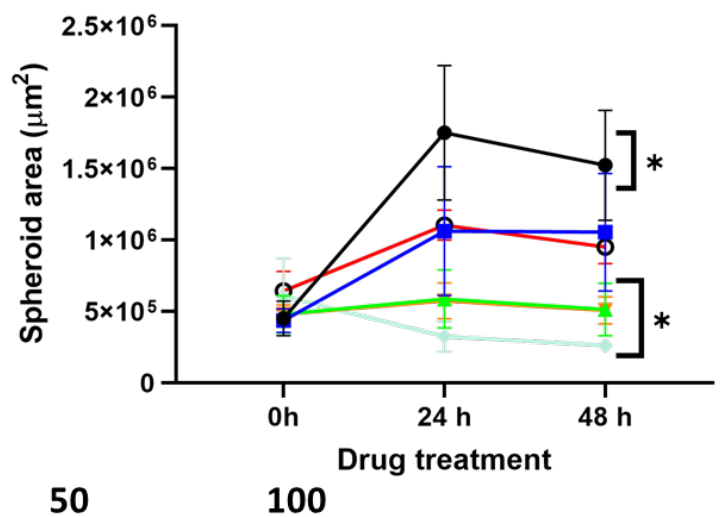

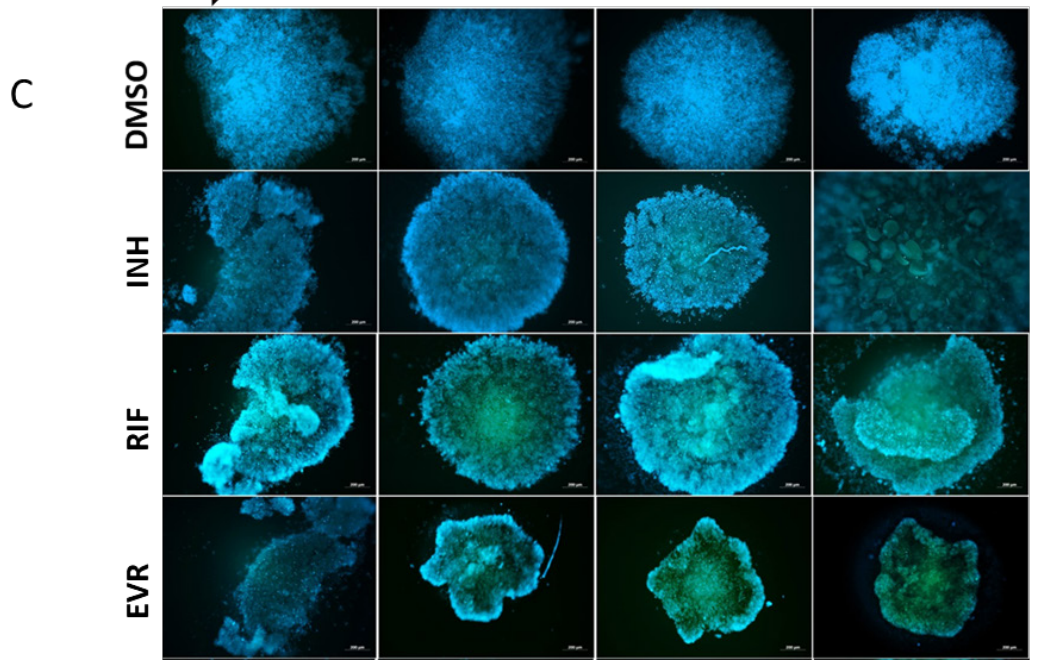

Figure 2: Evaluating drug response using Image cytometry. (a) mCherry intensity and (b) area for spheroids infected with BCG mCherry at MOI 100 quantification post drug treatment with control DMSO $(0.5 \% \mathrm{v} / \mathrm{v})$, single-drug treatment with isoniazid (INH $1.25 \mu M)$, rifampicin (RIF $0.195 \mu \mathrm{M}$ ), Everolimus (EVR 1nM) and combination of INH, RIF and EVR (0.625 $\mu \mathrm{M} \mathrm{INH,} 0.195 \mu \mathrm{M}$ RIF and $1 \mathrm{nM}$ EVR). * denotes statistical significance compared to DMSO at both 24 and $48 \mathrm{~h}$ time point using two-way Anova, $\mathrm{p}<0.05, \mathrm{n}=3$ samples/condition (c) Left to right shows increasing MOI of BCG and top to bottom shows Control, INH, RIF and EVR treated spheroids. Spheroids were stained with CYTO-ID autophagy dye (green) and Hoechst (blue).

pathway is upregulated upon Mtb infection and inhibiting the mTOR pathway increases host-cell autophagy and confer host-protective effects. Therefore, using mTOR pathway inhibition with EVR is expected to increase autophagy [43]. This supports the results observed in our model system where EVR treatment leads to an increase in host-cell autophagy.

\section{The effect of immune activation on TB spheroids}

To determine if monocyte activation influences spheroid formation and compactness, we used phorbol 12-myristate 13-acetate(PMA) to activateTHP-1 monocytes. Weidentified that activated spheroids (PMA treated) were compact when compared to the non-activated group as shown in Fig 3a. The spheroids were further quantified using Celigo image analysis software (Nexcelom Biosciences, MA), which demonstrated that the area of spheroids decreases with PMA treatment and monocyte activation (Fig 3b). In addition to the presence of tight structure, we validated the presence of physiochemical gradient using a $\mathrm{pH}$ probe. We observed that activated spheroids also depict a gradient in $\mathrm{pH}$ as shown in Fig S2 with a peripheral bright probe fluorescence (higher $\mathrm{pH}$; less acidic) and central lighter probe fluorescence (low $\mathrm{pH}$; more acidic). This gradient was absent in non-activated spheroids. To study the effect of PMA concentration on host cell viability and activation, we treated the spheroids with increasing concentrations of PMA ranging from $0-1000 \mathrm{ng} / \mathrm{ml}$ as shown in Figure 3c. To track host-cell viability/activation, we stained the 


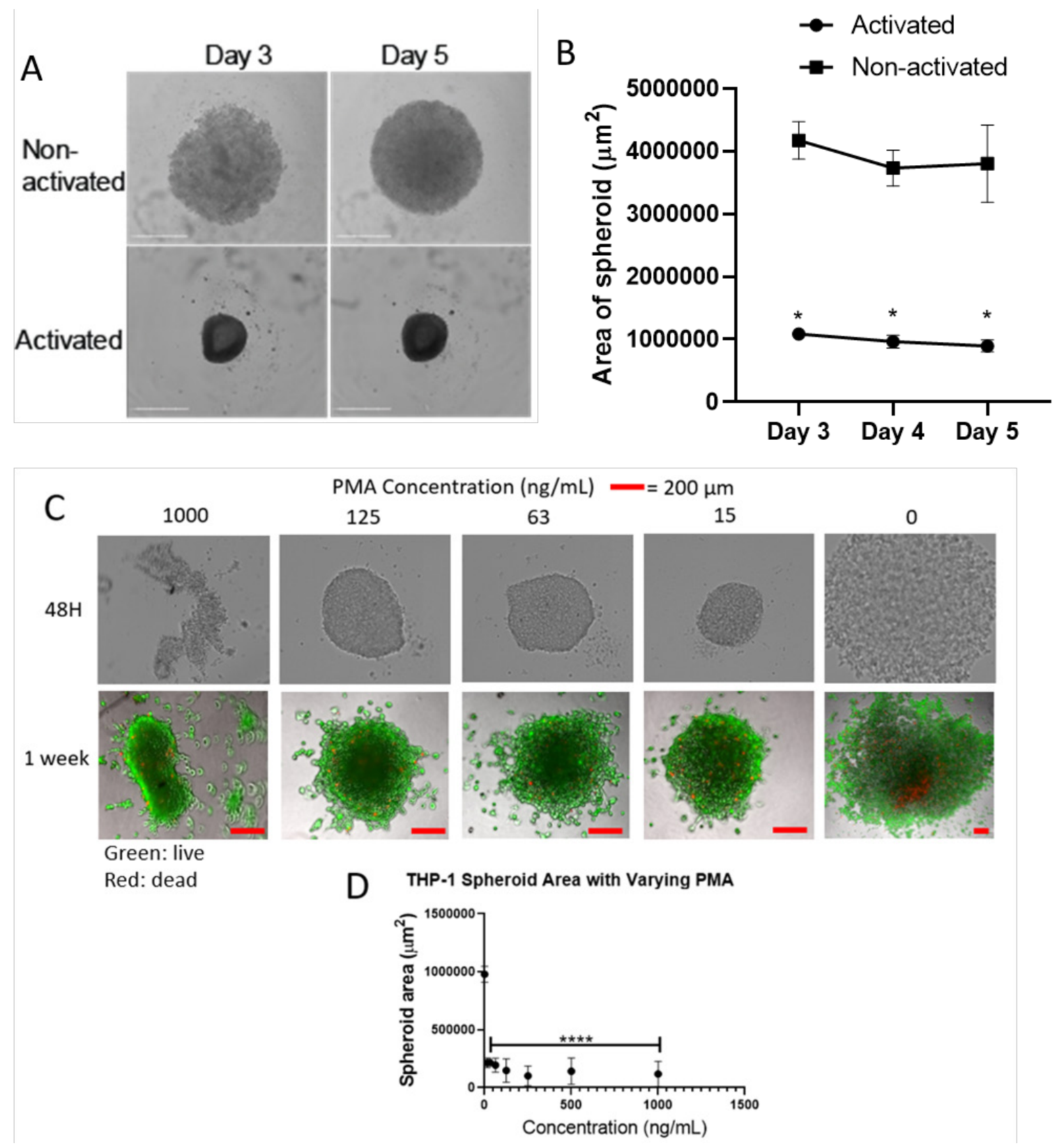

Figure 3: Evaluating the effects of monocyte activation on the formation of tight spheroids. (a) Bright field Images of non-activated (control) and PMA activated spheroids (b) Quantification of spheroid area. * denotes statistical significance at $p<0.05$ using one-way Anova test, $\mathrm{n}=3$ samples/condition (c) Images of spheroids treated with varying concentrations of PMA stained with Calcein AM (green-live) and

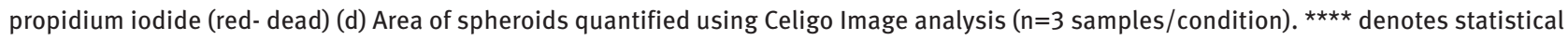
significance at $p<0.001$ when compared to the non-PMA treated group using One-way Anova and Tukey post-hoc test.

3D spheroids with Calcein AM (live cells) and propidium iodide (dead cells). We observed that there was no PMA concentration-dependent host-cell death (Fig 3c). Thus, monocyte activation was required for compaction of cells and formation of tight spheroids and did not influence host-cell viability.

\section{HIV induces mycobacterial proliferation and disrupts spheroid formation.}

After identifying that activation of monocytes was required for the formation of tight spheroids, we determined the effect of HIV-co-infection on TB spheroid formation and the bacteria present within the spheroids. 
We used THP-1 spheroids infected with BCG mCherry and HIV pseudotyped virus for studying the co-infection, and THP-1 spheroids without infection were included as a control. We chose THP-1, a human monocyte/macrophage cell line, because it supports Mtb infection similar to primary macrophages [44-46]. Additionally, macrophages are the major contributing cell type to the TB granuloma formation in vivo [47]. Therefore, we used THP-1 cells to model HIV-TB interactions in our studies.

Following the infection, TB The spheroids were tracked over 3 days, and mCherry intensity was quantified using Celigo software (Nexcelom Biosciences, MA) (Fig 4a). We observed an increase in BCG mCherry expression in the presence of HIV, indicating active bacterial proliferation (Fig 4b).Analysis of the cytokine levels in the supernatants of spheroid culture with or without infection revealed that HIV co-infection led to a significant increase in IL-1RA, IL-8, IP-10, MIP1- $\alpha$ and MIP1- $\beta$ when compared to no infection, HIV or BCG single infections (Fig 4c). In addition to this, we also found a complete disruption of spheroid formation with an increasing concentration of HIV pseudotype virus (Fig 4d). These results indicate that the spheroid model can depict structural aspects of HIV-TB infection.

\section{Discussion}

TB disease pathology is often characterized by the presence of granuloma, formed of macrophages engulfing Mtb and containing the infection. Significant efforts have been made towards capturing the complex cellularbacilli interactions in the TB field using several models ranging from PBMC aggregates, beads coated with Mtb antigens, microfluidic platforms and electro-spraying methods. The aspect of TB reactivation as a function of disrupted granuloma has been demonstrated with TNF- $\alpha$ inhibitors in 3D cell cultures [25,26]. Tereza et al., demonstrated the application of 3D granuloma model to study TB disease progression upon treatment with a PD-1 inhibitor [27]. However, the 3D cell culture granuloma mimetic has not been tested for HIV-TB studies. Here, we report the development of a simple THP-1 monocyte cell line-derived 3D spheroid model and establish infection with BCG mCherry. The use of fluorescently engineered mycobacteria has been reported previously by several studies $[48,49]$. The tagging of fluorescent probe enables easy tracking of bacterial localization and growth, as a function of intensity.

To determine the application of this model for anti-TB drug response, we used the fluorescent mCherry intensity measurements in response to known anti-TB compounds isoniazid and rifampicin and a host- a host-directed adjunctive therapeutic molecule which has been recently explored in the field of TB medicine [50]. We observed that a combination of INH, RIF and EVR leads to a maximum reduction of mCherry intensity, when compared to single drug treatments (Fig 2a). Similar results were observed by Ashley et al, where combinational drug treatment with INH and EVR led to higher bactericidal effect. Interestingly, we found that the size of the spheroid decreases when treated with EVR. It has been well reported in the cancer field that targeting mTOR pathway leads to shrinkage of tumors and it is very interesting to see similar shrinkage in size for TB spheroids[51]. We also observed the induction of autophagy with the drug treatment.

In this paper, we studied the role of immune cell activation using two approaches. In the first method (Fig 3\&4), we treated the host cells with PMA and identified that immune cell activation was necessary for the formation of tight spheroid structures (Fig 3a and b). The presence of tight structures induces the physicochemical gradient in $\mathrm{pH}$. Mature TB granulomas in humans, rabbits and NHPs are characterized by an acidic $\mathrm{pH}$ in the center or caseum[52]. To understand the role of depriving immune system function, we induced immunodeficiency within the 3D granulomas, by infecting with HIV, and found that mycobacteria can thrive and proliferate better in an immunocompromised microenvironment (Fig 3a, b). We also observed an increase in IL1-Ra, IL-8, IP-10, MIP1- $\alpha$ and $\beta$ levels in the culture supernatant of these spheroids. Previous studies have demonstrated that IL-1Ra [53] and IL-8 [54,55] are elevated in HIV-positive individuals. Similarly, IFN- $y$-inducible protein-10 (IP-10 or CXCL10), a chemoattractant for activated macrophages and $\mathrm{T}$ cells, increases with reactivation of LTBI and in HIV cases [56,57]. Chemokines MIP1- $\alpha$ and MIP1- $\beta$, involved in macrophage recruitment are elevated in the plasma of patients with HIV/TB [58]. Interestingly, we observed a complete disruption of granuloma formation in vitro with higher viral loads (Fig 4d). To our knowledge, this is the first in vitro 3D model to show the feasibility to synergistically study HIV-TB and demonstrate the structural integrity aspect of granulomas under HIV infections. Thus, our 3D spheroids models can aid in realtime tracking of granuloma formation and functionally analyze co-infections. Next generation spheroids can be engineered to include diverse cell population to mimic the microenvironment seen in TB patients. By incorporating a heterogeneous population of cells, this model can serve as an in vitro platform to study the effect of depletion of specific cell types in lung granulomas. 


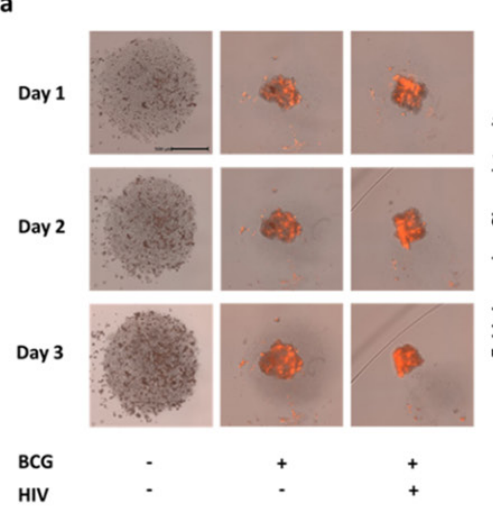

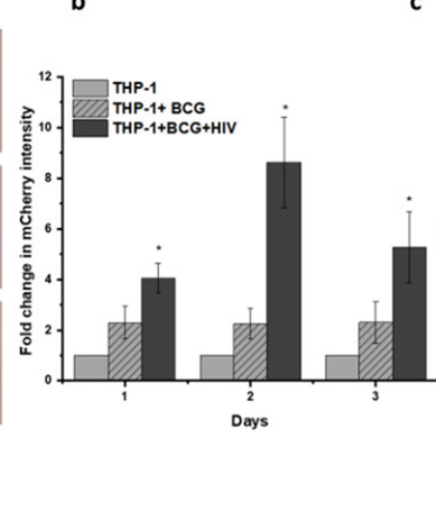

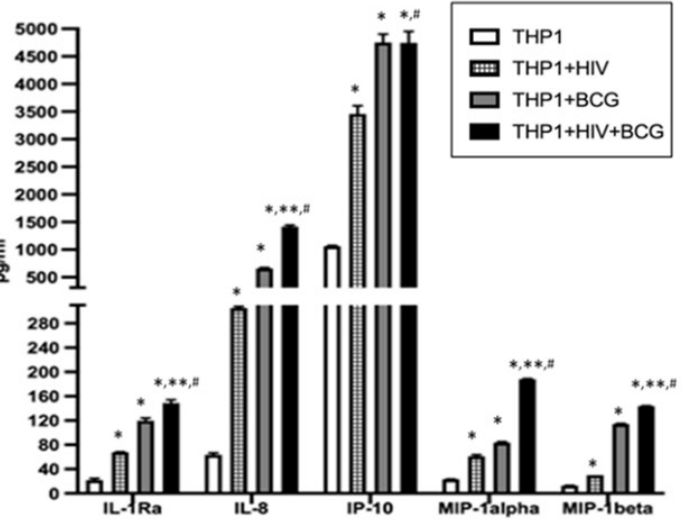

d

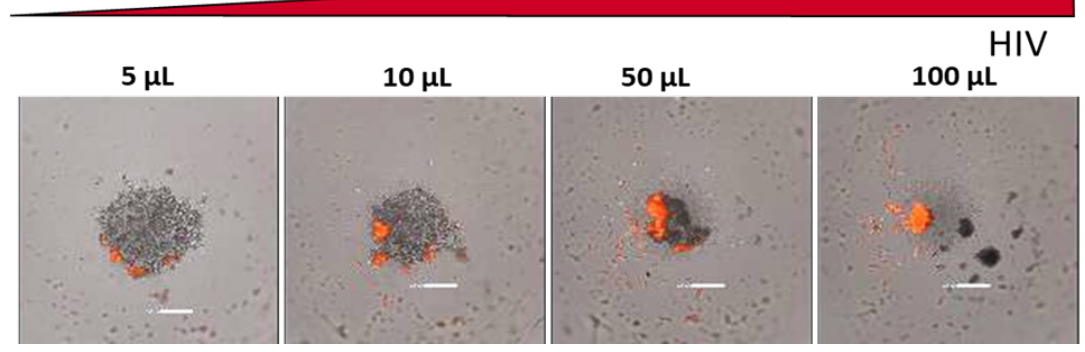

Figure 4: Modeling the interactions in HIV-TB infection and tracking mycobacteria using Image cytometry. a) Images of THP-1 spheroids generated by infecting 10,000 THP1 with BCG mCherry and HIV tracked over a period of 3 days. Data organized into 3 panels. Left to the right represents no infection control, BCG single infection group and HIV and BCG dual infection. Top to bottom represents increasing time in culture $(\mathrm{N}=3)$. (b) BCG mCherry proliferation by quantification of intensity over time. One-way Anova was used to determine statistical significance between BCG infected granulomas with and without HIV infection. * represents statistical significance, $p<0.05$, $(n=3)$ when compared to the THP-1 with BCG group. (c). Cytokine and chemokine levels in untreated vs. BCG and HIV-treated THP-1 spheroids assessed by multiplex cytokine analysis $(n=2)$. Paired two-tail T-test was used to determine significance between the sample groups. * denotes statistical significance when compared to THP-1 control group with no infection at $p<0.05$. **, \# represents the statistical significance of HIV-TB co-infection group when compared to BCG and HIV single infection respectively at $p<0.05$. (d) Images of THP-1 cell (Bright field) co-infected with BCG mCherry (Red) and HIV pseudotyped virus.

This platform is a proof-of-concept assay to show the establishment of high throughput 3D infection model to study TB pathogenesis in the context of HIV infection. Further, we demonstrate the development of quantitative image analysis techniques to characterize mycobacterialhost cell interactions in vitro. These endpoints can be adapted to establishing more complex primary immune cell-based spheroids, which are of greater significance to the TB field. The ability to measure spheroid parameters such as area provides an opportunity to identify the structural changes during early events of infection. The aspect of adding a dual infection by incorporating both HIV and mycobacterial infection in a single spheroid provides a unique ability to characterize the behavior of immune cells in response to co-infections. Thus, synergistic in vitro models can improve our understanding of TB-HIV interactions and develop better drug treatment regimens for HIV and TB co-infections. On the side of HIV research, a wide variety of cell-based, biochemical, and antibodybased high throughput in-vitro assays are available for the identification and evaluation of anti-retroviral compounds [59]. However, the HIV-TB co-infection poses a unique problem involving viral replication, TB-HIV drug-drug interactions, overlapping drug toxicities and tuberculosis-associated immune reconstitution inflammatory syndrome (IRIS). These factors impact the efficiency of ARTs to treat TB-HIV co-infection patients [60]. Therefore, this simple assay system can be used in the future to evaluate combinational anti-retroviral and anti-bacterial therapies in patient-derived peripheral blood mononuclear cells. In addition to this, the easy scaling capacity of this assay to 96 well plates can enable high throughput screens and the discovery of novel therapeutics for both TB and HIV infections. 
Author Contributions: SM, RB, TC, SS, BP conceptualized the idea. S.M developed the TB spheroid model and RB developed the spheroid-HIV co-infection model. SM, RB, TLC, SS, BP designed the experiments. SM, JL, RB, MT conducted the experiments. SS, TLC, BP provided overall project guidance and funding. S.M wrote the manuscript and all authors revised and approved.

Acknowledgement: This research was supported by grants awarded to R01EB012521 to BP and R01AI36948 to TLC by the National Institutes of Health.

Conflict of interest: Authors states no conflict of interest.

Data Availability Statement: The datasets generated during and analysed during the current study are available from the corresponding author on reasonable request.

\section{References}

1. Behr MA, Edelstein PH, Ramakrishnan L. Revisiting the timetable of tuberculosis. BMJ. 2018 Aug;362:k2738-2738.

2. Cohen A, Mathiasen VD, Schön T, Wejse C. The global prevalence of latent tuberculosis: a systematic review and meta-analysis. Eur Respir J. 2019 Sep;54(3):1900655.

3. Baluku JB, Anguzu G, Nassozi S, Babirye F, Namiiro S, Buyungo $R$, et al. Prevalence of HIV infection and bacteriologically confirmed tuberculosis among individuals found at bars in Kampala slums, Uganda. Sci Rep. 2020 Aug;10(1):13438.

4. Pawlowski A, Jansson M, Sköld M, Rottenberg ME, Källenius G. Tuberculosis and HIV co-infection. PLoS Pathog. 2012 Feb;8(2):e1002464-1002464.

5. Heuts F, Gavier-Widén D, Carow B, Juarez J, Wigzell H, Rottenberg ME. CD4+ cell-dependent granuloma formation in humanized mice infected with mycobacteria. Proc Natl Acad Sci USA. 2013 Apr;110(16):6482-7.

6. Nusbaum RJ, Calderon VE, Huante MB, Sutjita P, Vijayakumar S, Lancaster KL, et al. Pulmonary Tuberculosis in Humanized Mice Infected with HIV-1. Sci Rep. 2016 Feb;6(1):21522-21522.

7. Zhou D, Shen Y, Chalifoux L, Lee-Parritz D, Simon M, Sehgal PK, et al. Mycobacterium bovis bacille Calmette-Guérin enhances pathogenicity of simian immunodeficiency virus infection and accelerates progression to AIDS in macaques: a role of persistent T cell activation in AIDS pathogenesis. J Immunol. 1999 Feb;162(4):2204-16.

8. Zhou D, Lai X, Shen Y, Sehgal P, Shen L, Simon M, et al. Inhibition of adaptive Vgamma2Vdelta2+ T-cell responses during active mycobacterial coinfection of simian immunodeficiency virus SIVmac-infected monkeys. J Virol. 2003 Mar;77(5):2998-3006.

9. Mehra S, Golden NA, Dutta NK, Midkiff CC, Alvarez X, Doyle LA, et al. Reactivation of latent tuberculosis in rhesus macaques by coinfection with simian immunodeficiency virus. J Med Primatol. 2011 Aug;40(4):233-43.
10. Yuan T, Sampson NS. Hit Generation in TB Drug Discovery: From Genome to Granuloma. Chem Rev. 2018 Feb;118(4):1887-916.

11. Schaaf K, Hayley V, Speer A, Wolschendorf F, Niederweis M, Kutsch O, et al. A Macrophage Infection Model to Predict Drug Efficacy Against Mycobacterium Tuberculosis. Assay Drug Dev Technol. 2016 Aug;14(6):345-54.

12. Silva-Miranda M, Ekaza E, Breiman A, Asehnoune K, BarrosAguirre $\mathrm{D}$, Pethe $\mathrm{K}$, et al. High-content screening technology combined with a human granuloma model as a new approach to evaluate the activities of drugs against Mycobacterium tuberculosis. Antimicrob Agents Chemother. 2015 Jan;59(1):693-7.

13. Tezera LB, Bielecka MK, Chancellor A, Reichmann MT, Shammari $B A$, Brace $P$, et al. Dissection of the host-pathogen interaction in human tuberculosis using a bioengineered 3-dimensional model. eLife. 2017 Jan;6:e21283.

14. Elkington P, Lerm M, Kapoor N, Mahon R, Pienaar E, Huh D, et al. In Vitro Granuloma Models of Tuberculosis: potential and Challenges. J Infect Dis. 2019 May;219(12):1858-66.

15. Bielecka MK, Tezera LB, Zmijan R, Drobniewski F, Zhang X, Jayasinghe $S$, et al. A Bioengineered Three-Dimensional Cell Culture Platform Integrated with Microfluidics To Address Antimicrobial Resistance in Tuberculosis. MBio. 2017 Feb;8(1):e02073-16.

16. Berry SB, Gower MS, Su X, Seshadri C, Theberge AB. A Modular Microscale Granuloma Model for Immune-Microenvironment Signaling Studies in vitro. Front Bioeng Biotechnol. 2020 Aug;8(931):931.

17. Huang L, Kushner NL, Theriault ME, Pisu D, Tan S, McNamara CW, et al. The Deconstructed Granuloma: A Complex HighThroughput Drug Screening Platform for the Discovery of HostDirected Therapeutics Against Tuberculosis. Front Cell Infect Microbiol. 2018 Aug;8(275):275.

18. Yang D, Ding F, Mitachi K, Kurosu M, Lee RE, Kong Y. A Fluorescent Probe for Detecting Mycobacterium tuberculosis and Identifying Genes Critical for Cell Entry. Front Microbiol. 2016 Dec;7:20212021.

19. Manning AJ, Ovechkina Y, McGillivray A, Flint L, Roberts DM, Parish T. A high content microscopy assay to determine drug activity against intracellular Mycobacterium tuberculosis. Methods. 2017 Aug;127:3-11.

20. Tan S, Yates RM, Russell DG. Mycobacterium tuberculosis: Readouts of Bacterial Fitness and the Environment Within the Phagosome. Methods Mol Biol. 2017;1519:333-47.

21. Dartois V. The path of anti-tuberculosis drugs: from blood to lesions to mycobacterial cells. Nat Rev Microbiol. 2014 Mar;12(3):159-67.

22. Kiran D, Podell BK, Chambers M, Basaraba RJ. Host-directed therapy targeting the Mycobacterium tuberculosis granuloma: a review. Semin Immunopathol. 2016 Mar;38(2):167-83.

23. Fitzgerald LE, Abendaño N, Juste RA, Alonso-Hearn M; ThreeDimensional In Vitro Models of Granuloma to Study BacteriaHost Interactions, Drug-Susceptibility, and Resuscitation of Dormant Mycobacteria. Three-dimensional in vitro models of granuloma to study bacteria-host interactions, drugsusceptibility, and resuscitation of dormant mycobacteria. BioMed Res Int. 2014;2014:623856.

24. Bielecka MK, Tezera LB, Zmijan R, Drobniewski F, Zhang X, Jayasinghe $S$, et al. A bioengineered 3-dimensional cell culture 
platform integrated with microfluidics to address antimicrobial resistance in tuberculosis. MBio. 2017 Feb;8(1):e02073-16.

25. Mezouar S, Diarra I, Roudier J, Desnues B, Mege JL. Tumor Necrosis Factor-Alpha Antagonist Interferes With the Formation of Granulomatous Multinucleated Giant Cells: New Insights Into Mycobacterium tuberculosis Infection. Front Immunol. 2019 Aug;10:1947-1947.

26. Kapoor N, Pawar S, Sirakova TD, Deb C, Warren WL, Kolattukudy PE. Human granuloma in vitro model, for TB dormancy and resuscitation. PLoS One. 2013;8(1):e53657.

27. Tezera LB, Bielecka MK, Ogongo P, Walker NF, Ellis M, Garay-Baquero DJ, et al. Anti-PD-1 immunotherapy leads to tuberculosis reactivation via dysregulation of TNF- $\alpha$. eLife. 2020 Feb;9:e52668.

28. Bondalapati PK. A study on patients with TB and HIV co-infection in relation to mean CD4 counts. Indian Journal of Pharmacy Practice. 2017;10(2):111-4.

29. Huante MB, Nusbaum RJ, Endsley JJ. Co-Infection with TB and HIV: Converging Epidemics, Clinical Challenges, and Microbial Synergy. In: Cirillo JD, Kong Y, editors. Tuberculosis HostPathogen Interactions. Cham: Springer International Publishing; 2019. pp. 123-53.

30. Kwara A, Flanigan TP, Carter EJ. Highly active antiretroviral therapy (HAART) in adults with tuberculosis: current status. Int J Tuberc Lung Dis. 2005 Mar;9(3):248-57.

31. Blanc FX, Laureillard D, Goldfeld AE. Comment on: effects of time of initiation of antiretroviral therapy in the treatment of patients with HIV/TB co-infection, by Chelkeba L. et al. Ann Med Surg (Lond). 2020 Jul;57:22-3.

32. Chelkeba L, Fekadu G, Tesfaye G, Belayneh F, Melaku T, Mekonnen Z. Effects of time of initiation of antiretroviral therapy in the treatment of patients with HIV/TB co-infection: A systemic review and meta-analysis. Ann Med Surg (Lond). 2020 May;55:148-58.

33. Tasker C, Subbian S, Gao P, Couret J, Levine C, Ghanny S, et al. IFN- $\varepsilon$ protects primary macrophages against HIV infection. $\mathrm{JCI}$ Insight. 2016 Dec;1(20):e88255.

34. Dickinson JM, Aber VR, Mitchison DA. Bactericidal activity of streptomycin, isoniazid, rifampin, ethambutol, and pyrazinamide alone and in combination against Mycobacterium Tuberculosis. Am Rev Respir Dis. 1977 Oct;116(4):627-35.

35. Drusano GL, Sgambati N, Eichas A, Brown DL, Kulawy R, Louie A. The combination of rifampin plus moxifloxacin is synergistic for suppression of resistance but antagonistic for cell kill of Mycobacterium tuberculosis as determined in a hollow-fiber infection model. MBio. 2010 Aug;1(3):e00139-10.

36. Grosset J, Truffot-Pernot C, Lacroix C, Ji B. Antagonism between isoniazid and the combination pyrazinamide-rifampin against tuberculosis infection in mice. Antimicrob Agents Chemother. 1992 Mar;36(3):548-51.

37. Genestet C, Ader F, Pichat C, Lina G, Dumitrescu O, Goutelle S. Assessing the Combined Antibacterial Effect of Isoniazid and Rifampin on Four Mycobacterium tuberculosis Strains Using In Vitro Experiments and Response-Surface Modeling. Antimicrob Agents Chemother. 2017 Dec;62(1):e01413-7.

38. Ashley D, Hernandez J, Cao R, To K, Yegiazaryan A, Abrahem $R$, et al. Antimycobacterial Effects of Everolimus in a Human Granuloma Model. J Clin Med. 2020 Jun;9(7):2043.

39. Babu RR, Naresh K, Ravi A, Madhava Reddy B, Harinadha Babu V. Synthesis of novel isoniazid incorporated styryl quinazolinones as anti-tubercular agents against INH sensitive and MDR M. tuberculosis strains. Med Chem Res. 2014;23(10):4414-9.

40. Hassan ST, Gudomová M, Berchová-Bímová K, Gowrishankar S, Rengasamy KR. Antimycobacterial, Enzyme Inhibition, and Molecular Interaction Studies of Psoromic Acid in Mycobacterium tuberculosis: Efficacy and Safety Investigations. J Clin Med. 2018 Aug;7(8):E226.

41. Lohrasbi V, Talebi M, Bialvaei AZ, Fattorini L, Drancourt M, Heidary $M$, et al. Trends in the discovery of new drugs for Mycobacterium tuberculosis therapy with a glance at resistance. Tuberculosis (Edinb). 2018 Mar;109:17-27.

42. Kim JJ, Lee HM, Shin DM, Kim W, Yuk JM, Jin HS, et al. Host cell autophagy activated by antibiotics is required for their effective antimycobacterial drug action. Cell Host Microbe. 2012 May;11(5):457-68.

43. Cerni S, Shafer D, To K, Venketaraman V. Investigating the Role of Everolimus in mTOR Inhibition and Autophagy Promotion as a Potential Host-Directed Therapeutic Target in Mycobacterium tuberculosis Infection. J Clin Med. 2019 Feb;8(2):E232.

44. Bai X, Kim SH, Azam T, McGibney MT, Huang H, Dinarello CA, et al. IL-32 is a host protective cytokine against Mycobacterium tuberculosis in differentiated THP-1 human macrophages. J Immunol. 2010 Apr;184(7):3830-40.

45. Riendeau CJ, Kornfeld H. THP-1 cell apoptosis in response to Mycobacterial infection. Infect Immun. 2003 Jan;71(1):254-9.

46. Stokes RW, Doxsee D. The receptor-mediated uptake, survival, replication, and drug sensitivity of Mycobacterium tuberculosis within the macrophage-like cell line THP-1: a comparison with human monocyte-derived macrophages. Cell Immunol. 1999 Oct;197(1):1-9.

47. McClean CM, Tobin DM. Macrophage form, function, and phenotype in mycobacterial infection: lessons from tuberculosis and other diseases. Pathog Dis. 2016 Oct;74(7):ftw068. https:// doi.org/10.1093/femspd/ftw068.

48. Abramovitch RB. Mycobacterium tuberculosis Reporter Strains as Tools for Drug Discovery and Development. IUBMB Life. 2018 Sep;70(9):818-25.

49. MacGilvary NJ, Tan S. Fluorescent Mycobacterium tuberculosis reporters: illuminating host-pathogen interactions. Pathog Dis. 2018 Apr;76(3): https://doi.org/10.1093/femspd/fty017.

50. Singh $P$, Subbian $S$. Harnessing the mTOR Pathway for Tuberculosis Treatment. Front Microbiol. 2018 Jan;9:70.

51. Hua H, Kong Q, Zhang H, Wang J, Luo T, Jiang Y. Targeting mTOR for cancer therapy. J Hematol Oncol. 2019 Jul;12(1):71-71.

52. Via LE, Lin PL, Ray SM, Carrillo J, Allen SS, Eum SY, et al. Tuberculous granulomas are hypoxic in guinea pigs, rabbits, and nonhuman primates. Infect Immun. 2008 Jun;76(6):233340.

53. Takebe N, Paredes J, Pino MC, Lownsbury WH, Agosti J, Krown SE. Phase I/II trial of the type I soluble recombinant human interleukin-1 receptor in HIV-1-infected patients. J Interferon Cytokine Res. 1998 May;18(5):321-6.

54. Matsumoto T, Miike T, Nelson RP, Trudeau WL, Lockey RF, Yodoi J. Elevated serum levels of IL-8 in patients with HIV infection. Clin Exp Immunol. 1993 Aug;93(2):149-51.

55. Meddows-Taylor S, Martin DJ, Tiemessen CT. Dysregulated production of interleukin-8 in individuals infected with human immunodeficiency virus type 1 and Mycobacterium tuberculosis. Infect Immun. 1999 Mar;67(3):1251-60. 
56. Azzurri A, Sow OY, Amedei A, Bah B, Diallo S, Peri G, et al. IFN-ץinducible protein 10 and pentraxin 3 plasma levels are tools for monitoring inflammation and disease activity in Mycobacterium tuberculosis infection. Microbes Infect. 2005 Jan;7(1):1-8.

57. Juffermans NP, Verbon A, van Deventer SJ, van Deutekom H, Belisle JT, Ellis ME, et al. Elevated chemokine concentrations in sera of human immunodeficiency virus (HIV)-seropositive and HIV-seronegative patients with tuberculosis: a possible role for mycobacterial lipoarabinomannan. Infect Immun. 1999 Aug;67(8):4295-7.

58. Hanna LE, Bose JC, Nayak K, Subramanyam S, Swaminathan S. Short communication: influence of active tuberculosis on chemokine and chemokine receptor expression in HIV-infected persons. AIDS Res Hum Retroviruses. 2005 Dec;21(12):9971002.

59. Rumlová M, Ruml T. In vitro methods for testing antiviral drugs. Biotechnol Adv. 2018 May - Jun;36(3):557-76.

60. Mcllleron H, Meintjes G, Burman WJ, Maartens G. Complications of antiretroviral therapy in patients with tuberculosis: drug interactions, toxicity, and immune reconstitution inflammatory syndrome. J Infect Dis. 2007 Aug;196(s1 Suppl 1):S63-75. 